\title{
Distribution of ions around a charged sphere
}

\author{
P. Strating and F.W. Wiegel \\ Center for Theoretical Physics, Department of Applied Physics, Twente University, \\ P.O. Box 217, 7500 AE Enschede, The Netherlands
}

Received 15 September 1992

\begin{abstract}
Using a new method of calculating the effects of excluded volume, the authors evaluate the distribution of counterions around a charged sphere in thermal equilibrium. In regions of high concentration a saturation of the ion density is found, which is absent in the more conventional Gouy-Chapman model. At the same time the saturation effect of the potential (a property of the Gouy-Chapman model) is removed, giving significant corrections to the values of the potential and concentration at the surface of the sphere.
\end{abstract}

\section{Introduction}

The distribution of counterions around a central charge in a solvent is a well-known topic of theoretical research in chemistry and physics. A diffuse layer of ions, shielding the central charge, is one of the results of the Gouy-Chapman model, which is also known for its linearization according to Debye-Hückel, see for instance ref. [1], ch. 4.

In real physical systems the counterions are not point particles, and to find realistic results it is necessary to study the influence of steric hindrance on the counterion distribution. The steric hindrance inhibits the counterion concentration from exceeding a saturation value of $a^{-3}$, where $a^{3}$ is the effectively blocked (excluded) volume of a single ion. One expects the steric hindrance to be important in regions of high concentration, i.e. close to the central charge.

To study the main effect of excluded volume on the charge distribution we use a method recently developed by one of the authors [2]. In order to stay close to the Debye-Hückel results, we will study the charge distribution around a central sphere of radius $R$ and total charge $Q$. The sphere is immersed in a solvent with relative dielectric constant $\varepsilon$. Each counterion carries a charge $q$ such that $q Q<0$, and has an effective excluded volume $a^{3}$. At infinity the concentration of ions is $c_{0}$, and to obtain neutrality at infinity we assume the presence of a uniform background charge field of strength $-q c_{0}$ everywhere outside the sphere. Note that in the usual Gouy-Chapman model, 
the neutrality at infinity is obtained through the presence of a second kind of movable counterions with opposite charge $-q$. We have chosen to study the problem of only one kind of counterions for reasons of simplicity, but intend to tackle the more complicated problem in future.

We want to calculate the counterion concentration $c(r)$ in thermal equilibrium. Because of the spherical symmetry, the concentration - or equivalently the electrostatic potential $\varphi(r)$ - only depends on the radial distance $r$ from the center of the sphere.

\section{Basic equations}

In the method of ref. [2], the diffusion coefficient $D_{0}$ (given by $k_{\mathrm{B}} T / f_{\mathrm{I}}$ with $f_{\mathrm{T}}$ the translational coefficient of friction, $k_{\mathrm{B}}$ Boltzmann's constant and $T$ the temperature) is replaced by a concentration dependent diffusion coefficient $D(c)$,

$$
D(c)=\frac{D_{0}}{1-a^{3} c} .
$$

We are now able to write down the basic equations. The local particle current density is given by

$$
j(r)=-D(c) \nabla c+\frac{F}{f_{\mathrm{I}}} c(\boldsymbol{r}),
$$

where $\boldsymbol{F}$ is the force on a single counterion, given by $q E(\boldsymbol{r}) . E(r)$ is the local electrostatic field. In thermal equilibrium, $j$ should vanish identically and using the spherical symmetry, $E=-\nabla \varphi$ and eq. (1), we find

$$
\frac{\mathrm{d} \varphi}{\mathrm{d} r}=-\frac{k_{\mathrm{B}} T}{q} \frac{1}{c\left(1-a^{3} c\right)} \frac{\mathrm{d} c}{\mathrm{~d} r} .
$$

In addition to this equation which relates the electrostatic potential $\varphi(r)$ to $c(r)$, we use the Maxwell relation $\operatorname{div} \varepsilon E=4 \pi \rho$. As $E=-\nabla \varphi$ we have $\Delta \varphi=-4 \pi \rho / \varepsilon$ (we use Gaussian units in which $\varepsilon_{0}=1$, see for instance ref. [3]), with the charge density $\rho(r)=q c(r)-q c_{0}$ outside the sphere. This gives us a second relation between $\varphi$ and $c$, which reads in spherical coordinates:

$$
\left(\frac{\mathrm{d}}{\mathrm{d} r}+\frac{2}{r}\right) \frac{\mathrm{d} \varphi}{\mathrm{d} r}=-\frac{4 \pi q}{\varepsilon}\left[c(r)-c_{0}\right] .
$$

By combining eqs. (3) and (4) we arrive at our fundamental equation for the equilibrium concentration, 


$$
\left(\frac{\mathrm{d}}{\mathrm{d} r}+\frac{2}{r}\right) \frac{1}{c\left(1-a^{3} c\right)} \frac{\mathrm{d} c}{\mathrm{~d} r}=\frac{4 \pi q^{2}}{k_{\mathrm{B}} T \varepsilon}\left[c(r)-c_{0}\right], \quad r>R .
$$

Leaving out the factor $1-a^{3} c$ in the denominator would reduce the equation to the usual equation without excluded volume.

We now write this equation in a dimensionless form by introducing the new variables $f=a^{3} c, f_{0}=a^{3} c_{0}$ and $x=\left(x_{0} / R\right) r$, with

$$
x_{0}=R \sqrt{\frac{4 \pi q^{2}}{k_{\mathrm{B}} T a^{3} \varepsilon}},
$$

to obtain for $x \geqslant x_{0}$

$$
\left(\frac{\mathrm{d}}{\mathrm{d} x}+\frac{2}{x}\right) \frac{1}{f(1-f)} \frac{\mathrm{d} f}{\mathrm{~d} x}=f(x)-f_{0} .
$$

We can also write the problem in an alternative formulation by using the function $g=\ln (f / 1-f)$. Then

$$
\left(\frac{\mathrm{d}}{\mathrm{d} x}+\frac{2}{x}\right) \frac{\mathrm{d} g}{\mathrm{~d} x}=\frac{\mathrm{e}^{g}}{1+\mathrm{e}^{g}}-f_{0} .
$$

It is easily seen by integration of eq. (3) and the boundary condition for $f$ that

$$
g=-\frac{q \varphi}{k_{\mathrm{B}} T}+\ln \frac{f_{0}}{1-\int_{0}}
$$

as $\varphi$ is supposed to vanish for large $r$. So $g$ is a dimensionless potential. We have now rewritten the functions $c(r)$ and $\varphi(r)$ in terms of dimensionless functions $f(x)$ and $g(x)$.

Besides the boundary condition at infinity we need to have a second boundary condition to incorporate the charge of the central sphere and to find a unique solution. Therefore we integrate the Maxwell equation $\Delta \varphi=-4 \pi \rho / \varepsilon$ over the volume $V_{R}$ of the sphere with radius $R$ and use Gauss' theorem to obtain

$$
\int_{V_{R}} \Delta \varphi(r) \mathrm{d} r=\left.4 \pi R^{2} \frac{\mathrm{d} \varphi}{\mathrm{d} r}\right|_{r=R}=-\frac{4 \pi Q}{\varepsilon},
$$

which gives us the desired boundary condition. In our four equivalent formulations 


$$
\begin{aligned}
& \left.\frac{\mathrm{d} \varphi}{\mathrm{d} r}\right|_{r=R}=-\frac{Q}{\varepsilon R^{2}},\left.\quad \frac{1}{c\left(1-a^{3} c\right)} \frac{\mathrm{d} c}{\mathrm{~d} r}\right|_{r=R}=\frac{q Q}{k_{\mathrm{B}} T \varepsilon R^{2}}, \\
& \left.\frac{\mathrm{d} g}{\mathrm{~d} x}\right|_{x=x_{0}}=-\frac{\mu}{x_{0}},\left.\quad \frac{1}{f(1-f)} \frac{\mathrm{d} f}{\mathrm{~d} x}\right|_{x=x_{0}}=-\frac{\mu}{x_{0}},
\end{aligned}
$$

where we introduced the dimensionless parameter $\mu=-q Q / k_{\mathrm{B}} T \varepsilon R$, which is always greater than zero and measures the competition between the electrostatic potential energy and the thermal energy.

\section{The Debye-Hückel limit}

In the Debye-Hückel theory one linearizes the nonlinear differential equation for $\varphi(r)$ in the region where $\left|q \varphi / k_{\mathrm{B}} T\right| \ll 1$, which may be satisfied everywhere outside the central sphere, but usually only holds for $r \rightarrow \infty$.

To obtain the nonlinear equation for $\varphi$, we write eq. (8) in terms of $\varphi$ using eq. (9), yielding

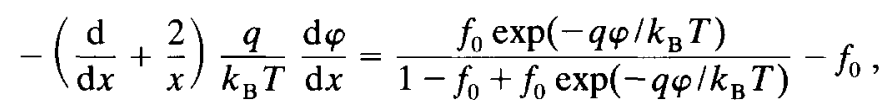

and after linearization with respect to $q \varphi / k_{\mathrm{B}} T$ we are left with

$$
\left(\frac{\mathrm{d}}{\mathrm{d} x}+\frac{2}{x}\right) \frac{\mathrm{d} \varphi}{\mathrm{d} x}=\left(f_{0}-f_{0}^{2}\right) \varphi .
$$

This equation can be solved exactly to give the asymptotic solution for $\varphi$ (the $\sim$ denotes proportionality):

$$
\varphi(x) \sim \frac{\exp \left(-\sqrt{f_{0}-f_{0}^{2}} x\right)}{x} \text { or } \varphi(r) \sim \frac{\exp (-\kappa r)}{r},
$$

with the Debye-Hückel inverse screening length $\kappa$ given by

$$
\kappa=\frac{x_{0}}{R} \sqrt{f_{0}-f_{0}^{2}}=\sqrt{\frac{4 \pi q^{2}}{k_{\mathrm{B}} T a^{3} \varepsilon}\left(f_{0}-f_{0}^{2}\right)} .
$$

The $a^{3}$-dependence disappears only if we can neglect $f_{0}^{2}$ compared to $f_{0}$ and insert $f_{0}=a^{3} c_{0}$ in eq. (15). Because $f_{0}$ is a volume fraction, we always have $f_{0}^{2} \ll f_{0}$ if the blocked volume $a^{3}$ goes to zero. Our result for $\kappa$ differs by a factor $\sqrt{2}$ from the regular Debye-Hückel theory because we have used a 
uniform background charge, in contrast to the two kinds of moveable counterions in the usual model.

We conclude this section by noting that for very small ratios of $\mu / x_{0}$, which means that the potential energy is small compared to the thermal energy even close to the sphere, the asymptotic solution will be approximately valid for all $x$. Using the boundary condition for $r=R$ we find the approximate solution

$$
\varphi(r) \approx \frac{Q}{\varepsilon(1+\kappa R)} \frac{\mathrm{e}^{-\kappa(r-R)}}{r}
$$

from which the potential strength at the surface of the spherc can be calculated directly.

\section{Properties of the solution and numerical results}

Before turning to numerical integration methods we discuss some general properties of the solution of eq. (7). We restrict ourselves to the physical solutions and do not attempt to give an exhaustive analysis of the nonlinear differential equation.

The primary physical condition for $f$ is that $f(x) \geqslant 0$ for all $x \geqslant x_{0}$. Moreover, we demand that $f$ and $g$ be well-behaved for all $x \geqslant x_{0} ; g$ because it is related to the physical potential $\varphi$. From these demands we derive some properties of $f$.

First, the function $f$ can never assume the values 0 or 1 for finite $x$, because if it did, $g$ would not exist at that point (recall that $g=\ln [f /(1-f)]$ ), contrary to our demand of regularity for $g$. Therefore, as we know that at least at infinity $0<f<1$, we conclude $0<f(x)<1$ for all $x \geqslant x_{0}$.

Second, the derivative of $f$ is never zero for finite $x$. To see this, we need a little more work and rewrite eq. (7) by writing out the differentiations on the lefthand side,

$$
\frac{2 f-1}{f^{2}(1-f)^{2}}\left(\frac{\mathrm{d} f}{\mathrm{~d} x}\right)^{2}+\frac{1}{f(1-f)} \frac{\mathrm{d}^{2} f}{\mathrm{~d} x^{2}}+\frac{2}{x f(1-f)} \frac{\mathrm{d} f}{\mathrm{~d} x}=f-f_{0} .
$$

If we put $\mathrm{d} f / \mathrm{d} x=0$ for $x=\bar{x}$, we find that at a minimum or maximum $f$ should obey

$$
\left.\frac{1}{f(1-f)} \frac{\mathrm{d}^{2} f}{\mathrm{~d} x^{2}}\right|_{x=\bar{x}}=f(\bar{x})-f_{0} .
$$

As $f(1-f)>0$ for all $\bar{x}$, there are now three possibilities: we always have a minimum at $\bar{x}$ if $f(\bar{x})>f_{0}$, a maximum if $f(\bar{x})<f_{0}$, and an undetermined 
extremum if $f(\bar{x})=f_{0}$. The first two cases cannot happen: $f$ would never be able to meet its asymptotic boundary condition, because this would require an additional extremum. The third possibility is that $\mathrm{d} f / \mathrm{d} x(\bar{x})=0$ and at the same time $f(\bar{x})=f_{0}$. However, an inflection point is not possible for the same reason as mentioned above. But there is a possibility that $f$ reaches $f_{0}$ at $\bar{x}$ with zero derivative, and never leaves the value $f_{0}$ again. We now show that this case too can never occur, by trying to find an $f$ that does satisfy these requirements. For, knowing $f(1-f) \neq 0$, we can conclude from eq. (3) that

$$
\frac{\mathrm{d} f}{\mathrm{~d} x}(\bar{x})=0 \Leftrightarrow \frac{\mathrm{d} \varphi}{\mathrm{d} x}(\bar{x})=0
$$

and invoke eq. (9) to find that $f(\bar{x})=f_{0}$ implies $\varphi(\bar{x})=0$. So if we are trying to find an $f$ satisfying $f(\bar{x})=f_{0}$ and $\mathrm{d} f / \mathrm{d} x(\bar{x})=0$ this is equivalent to finding a $\varphi$ for which $\varphi(\bar{x})=\mathrm{d} \varphi / \mathrm{d} x(\bar{x})=0$. But such a solution, if it exists, can be found by linearization around $x=\bar{x}$, cf. section 3 . Following the same linearization procedure, one finds that the only solution satisfying the conditions at $x=\bar{x}$ is that $\varphi=0$ for all $x$ near $\bar{x}$. The region of validity of the linearization can now be enlarged, to find that $\varphi=0$ in this larger region, and so on. This leads to the solution $\varphi=0$ for all $x \geqslant \bar{x}$, which can be translated to $f(x)=f_{0}$ for all $x$. But $f(x)=f_{0}$ is not a solution of our boundary value problem, because it does not satisfy the boundary condition at $x_{0}$. Therefore $f$ cannot have a zero derivative above the $f_{0}$-line, on the $f_{0}$-line or under the $f_{0}$-line, so $f$ cannot have a zero derivative at all for finite $x$.

Because the derivative at $x_{0}$ is smaller than zero, we conclude that $f$ is a monotonically decreasing function, and that $f_{0}<f<1$.

In fig. 1 we have plotted the solutions of eq. (7) for various boundary conditions. The solutions were calculated using fourth and fifth order RungeKutta algorithms. It is clearly visible that while the solution for $f$ is extremely unexciting for $\mu / x_{0} \leqslant 1$, it develops a plateau close to the sphere for larger ratios of $\mu / x_{0}$. So when the attractive force of the central sphere is strong compared to the diffusive force, there is a region of saturation with close packing concentration $c=a^{-3}$.

In fig. 2 we have plotted the corresponding solutions $g(x)$ of eq. (8). Note that we have normalized $g$ to zero at infinity, thus turning $g$ into a true dimensionlesss potential given by $-q \varphi / k_{\mathrm{B}} T$. Table I gives some values of this normalized $g\left(x_{0}\right)$ for different values of $\mu / x_{0}$. From these values we see that the dimensionless potential does not show saturation as known from the ordinary Gouy-Chapman model. This is easily explained, as the concentration of counterions does saturate and is not capable anymore of compensating high potential values by heaping up near the sphere. 


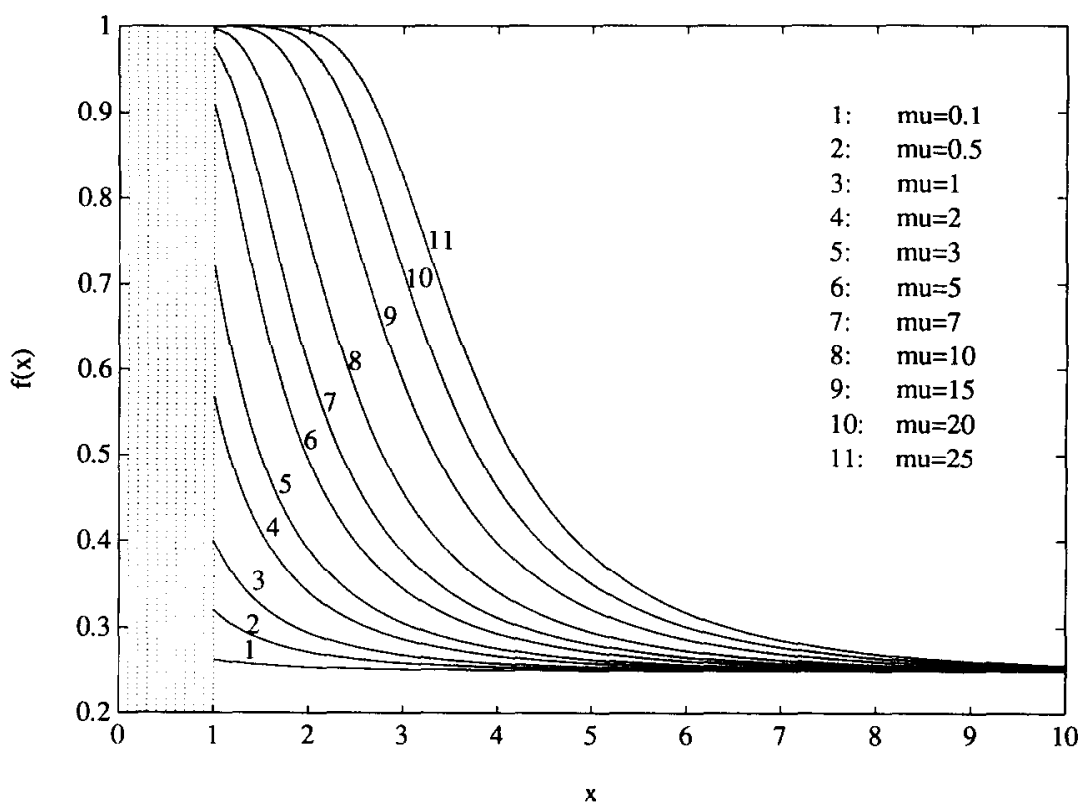

Fig. 1. The concentration $f$ as a function of $x\left(x_{0}=1, f_{0}=0.25\right)$.

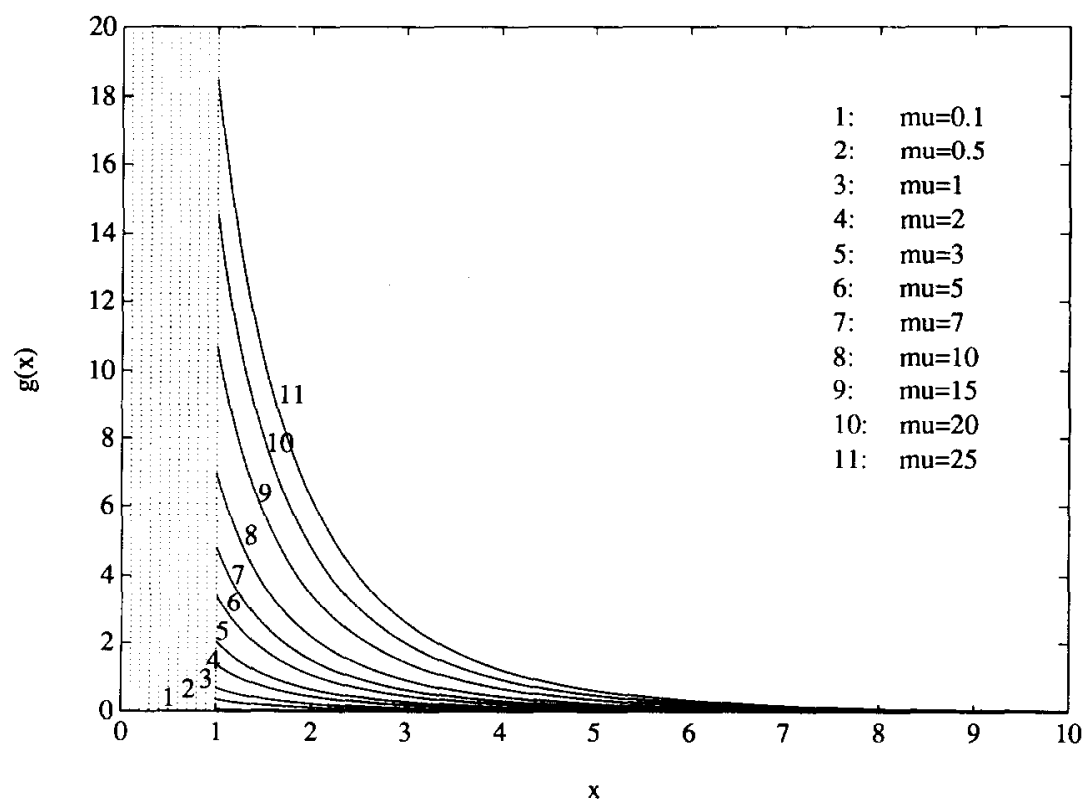

Fig. 2. The dimensionless potential $g$ as a function of $x\left(x_{0}=1, f_{0}=0.25\right)$. 
Table I

Potential values at the surface of the sphere $\left(x_{0}=1, f_{0}=0.25\right)$.

\begin{tabular}{llllllllll}
\hline$\mu$ & $g\left(x_{0}\right)$ & $\mu$ & $g\left(x_{0}\right)$ & $\mu$ & $g\left(x_{0}\right)$ & $\mu$ & $g\left(x_{0}\right)$ & $\mu$ & $g\left(x_{0}\right)$ \\
\hline 0.1 & 0.07 & 1 & 0.69 & 3 & 2.05 & 7 & 4.80 & 15 & 10.7 \\
0.5 & 0.35 & 2 & 1.37 & 5 & 3.41 & 10 & 6.96 & 25 & 18.5 \\
\hline
\end{tabular}

Table II

Potential values at the surface of the sphere for different concentrations at infinity $\left(x_{0}=1\right.$, $\mu=3)$.

\begin{tabular}{lllllll}
\hline$f_{0}$ & 0.01 & 0.10 & 0.25 & 0.45 & 0.60 & 0.75 \\
$g\left(x_{0}\right)$ & 2.66 & 2.21 & 2.05 & 2.02 & 2.06 & 2.17 \\
\hline
\end{tabular}

Apart from their dependence on $\mu / x_{0}$, the solutions $f$ and $g$ also depend on $f_{0}$, the concentration at infinity, but only quantitatively. The qualitative behaviour is the same for all $f_{0}<1$. We only note that for higher $f_{0}$ the saturation effect is reached for smaller $\mu$. Table II gives some values of $g\left(x_{0}\right)$ for different $f_{0}$ but with $\mu / x_{0}$ kept fixed at a value of 3 .

\section{Conclusions}

The Gouy-Chapman model was developed to describe the diffuse layer near a charged surface. This charged surface itself is supposed to be a compact layer of immobilized ions attached to an attractor, called the Stern layer. The present method suggests that we do not need these presumptions anymore, because it is possible to extend the Gouy-Chapman model into the Stern layer up to the attractor underneath it.

In this simple, classical problem we have demonstrated the strength of the present method to handle problems in which excluded volume plays a role. We have seen that the solutions behave most regularly, satisfying all physical expectations. However, it will not be easy to test the quantitative results experimentally, because of the rather unrealistic uniform background charge field, but the results may provide insight in the structure of the diffuse layer near the sphere.

\section{References}

[1] W.B. Russell, D.A. Saville and W.R. Schowalter, Colloidal Dispersions (Cambridge Univ. Press, Cambridge, 1989).

[2] F.W. Wiegel, Physical Principles in Chemoreception, Lecture Notes in Biomathematics (Springer, Berlin, 1991).

[3] J.D. Jackson, Classical Electrodynamics, 2nd ed. (Wiley, New York, 1975). 